\title{
Assessing the extent of non-aggressive cancer in clinically detected stage I non-small cell lung cancer
}

\author{
Minal S Kale, ${ }^{1}$ Keith Sigel, ${ }^{1}$ Grace Mhango, ${ }^{1}$ Juan P Wisnivesky ${ }^{1,2}$
}

${ }^{1}$ Division of General Internal Medicine, Department of Medicine, Icahn School of Medicine at Mount Sinai, New York City, New York, USA ${ }^{2}$ Division of Pulmonary, Critical Care, and Sleep Medicine, Icahn School of Medicine at Mount Sinai, New York City, New York, USA

\section{Correspondence to} Dr Minal S Kale, Division of General Internal Medicine, Department of Medicine, Icahn School of Medicine at Mount Sinai, New York, NY 10029, USA;

minal.kale@mountsinai.org

Received 23 March 2017 Revised 29 August 2017

Accepted 25 September 2017 Published Online First 20 October 2017

\section{ABSTRACT}

Background Overdiagnosis among clinically detected lung cancers likely consists of cases that are nonaggressive and slowly progressive and will never disseminate, cause symptoms or be a threat to a subject's survival, even if untreated. In this study, we estimate the prevalence of non-aggressive lung cancers from a large, population-based cancer registry.

Methods We identified individuals $\geq 65$ years with histologically confirmed, untreated stage I non-small cell lung cancers (NSCLCs) from the Surveillance, Epidemiology, and End Results-Medicare registry. We estimated the rate of non-aggressive lung cancers by determining the point at which the cumulative lung cancer-specific survival curve no longer changed (ie, the slope approaches zero). At this point, there are no additional deaths due to progressive lung cancer observed among untreated patients after adjusting for deaths from competing risks (these long-term survivors can be considered 'non-aggressive cases).

Results The overall rate of non-aggressive cancers among 2197 clinically detected cases of untreated stage I NSCLC was $2.4 \%, 95 \% \mathrm{Cl}: 1.0 \%$ to $3.8 \%$. The rate of non-aggressive cancer was $1.9 \%(95 \% \mathrm{Cl}: 0.0 \%$ to $4.9 \%)$ for women and $2.4 \%$ (95\% Cl: $0.7 \%$ to $4.1 \%)$ for men $(p=0.84)$. When stratifying by tumour size, nonaggressive cancer rates were $10.2 \%(95 \% \mathrm{Cl}: 0.0 \%$ to $29.3 \%), 2.1 \%$ (95\% Cl: $0.0 \%$ to $9.2 \%), 4.9 \%(95 \% \mathrm{Cl}$ : $0.0 \%$ to $10.3 \%), 1.8 \%(95 \% \mathrm{Cl}: 0.0 \%$ to $5.2 \%)$ and $0.0 \%$ (95\% Cl: $0.0 \%$ to $1.0 \%$ ) for tumour sizes $<15$ $\mathrm{mm}, 15-24 \mathrm{~mm}, 25-34 \mathrm{~mm}, 35-44 \mathrm{~mm}$ and $\geq 45 \mathrm{~mm}$, respectively. In comparison with the smallest tumour sizes $(<15 \mathrm{~mm})$, the rates of non-aggressive cancers were not statistically significantly different for tumour sizes $15-24 \mathrm{~mm}(p=0.36), 25-34 \mathrm{~mm}(p=0.57), 35-44 \mathrm{~mm}$ $(p=0.38)$ and tumour sizes $>45 \mathrm{~mm}(p=0.30)$.

Discussion We found relatively low rates of nonaggressive cancers among clinically detected, stage I NSCLC regardless of sex or size. Our findings suggest that most clinically diagnosed early stage cancers should be treated with curative intent.

\section{INTRODUCTION}

In the context of medical screening, an 'overdiagnosed' cancer refers to a cancer which would never have been diagnosed in the patient's lifetime if screening had not taken place. ${ }^{1-3}$ However, a similar phenomenon also occurs among clinically detected cancers and consists of cancers that are non-aggressive and slowly progressive and will never disseminate, cause symptoms or be a threat to a subject's survival, even if untreated. It is likely

\section{Key messages}

What is the key question?

- What are the rates of non-aggressive cancers in stage I non-small cell lung cancer?

What is the bottom line?

- In a population-based cancer registry, there were low rates of non-aggressive cancers.

Why read on?

- We used a sound statistical method (competing risks) to assess rates of non-aggressive cancers and also conducted analyses stratified by age, gender, the presence of COPD, tumour size and histology.

that these cancers have been found incidentally via imaging for another purpose. The presence of clinically diagnosed, non-aggressive cancers may be influenced by certain patient characteristics, such as age and sex, as well as tumour characteristics, such as histology and tumour size, among other factors. Unfortunately, clinically diagnosed, non-aggressive cancer is difficult to ascertain at the time of diagnosis as there are no existing markers to differentiate aggressive versus non-progressive cancers. Treating these cancers potentially leads to unnecessary morbidity and mortality without a survival benefit.

The magnitude of non-aggressive cancers among non-screened lung cancers has previously been evaluated in observational cohorts. However, previous studies have been hindered by a failure to appropriately treat non-lung cancer deaths as a competing risk, leading to biased estimation of the extent of this issue. In the context of lung cancer screening, older studies evaluating the role of chest radiograph screening suggested that overdiagnosis may explain some of the excess cancers and stage shift observed among screened smokers. ${ }^{45}$ More recent modelling studies have estimated that approximately $1.5-6.6 \%$ of all cancers (including screen-detected cancers) are overdiagnosed. ${ }^{6}$ Analysis of data from the National Lung Screening Trial (NLST) has yielded estimates of overdiagnosis with a conservative estimate of $18.5 \%$ and a wide range of plausible values depending on the histological subtype and applied definition. ${ }^{7}$ Data from European lung cancer screening trials have also suggested that lung cancer screening leads to overdiagnosis. ${ }^{89}$

Understanding the true rate of non-aggressive cancers among clinically diagnosed lung cancers is 
critical for evaluating their management. In this study, we used competing risks methods to assess lung cancer-specific survival of untreated patients with stage I non-small cell lung cancer (NSCLC) to estimate the extent of non-aggressive cancer from a large, population-based cancer registry.

\section{METHODS}

We identified individuals from the Surveillance, Epidemiology, and End Results (SEER) registry linked to Medicare claims. ${ }^{10}$ SEER is a coordinated, national cancer registry that collects detailed cancer information covering approximately $28 \%$ of the US population and is nationally representative. ${ }^{11}$ Our sample consisted of patients $\geq 65$ years diagnosed with histologically confirmed, untreated stage I NSCLC diagnosed between 1992 and 2010. In order to ensure complete ascertainment of lung cancer treatments, we further limited our cohort to Medicare beneficiaries with both Part A (inpatient) and Part B (outpatient) coverage and excluded patients in health maintenance organisations as they lacked complete and detailed claim information. To reduce the likelihood that patients had undetected mediastinal lymph node involvement, we only included individuals with a claim for a diagnostic CT of the chest, positron emission tomography (PET)-CT or mediastinoscopy in the 30 days preceding and 90 days beyond the date of diagnosis.

Sociodemographic information (age, sex, race, ethnicity, marital status and estimated income) was obtained from the SEER and Medicare databases. To evaluate the presence of COPD status of individuals at the time of cancer diagnosis, we used a combination of inpatient, outpatient and physician claims. $^{12} 13$

SEER cancer coding provided information enabling ascertainment of tumour histology (adenocarcinoma, squamous cell carcinoma, large cell carcinoma or other). Additionally, cancer stage was assessed using detailed SEER information on tumour location, size, local extension and lymph node involvement. Tumour sizes were classified into the following five categories: $<15 \mathrm{~mm}$, $15-24 \mathrm{~mm}, 25-34 \mathrm{~mm}, 35-44 \mathrm{~mm}$ and $\geq 45 \mathrm{~mm}$. We defined stage I NSCLC based on the seventh edition of the American Joint Committee on Cancer system. ${ }^{14}$

We used Medicare claims to determine the diagnostic and staging workup of patients, including the use of CT, PET and mediastinoscopy. We evaluated the use of surgery, radiotherapy or chemotherapy using a combination of SEER and Medicare claims. Patients without evidence of claims or SEER data indicating the use of any of these strategies were classified as untreated and included in the cohort. SEER does not include robust information regarding the reason for lack of treatment of these individuals.

The primary study outcome, lung cancer-specific survival, was determined from SEER data, which have high levels of ascertainment. ${ }^{15}$ Cause of death (lung cancer vs non-lung cancer) was collected from SEER and was based on death certificate data. Survival time was determined as the interval from the date of diagnosis to the date of lung cancer death recorded by SEER. Subjects surviving past 140 months (to avoid estimating survival curves based on a small number of cases) or 31 December 2010 were censored.

\section{STATISTICAL ANALYSIS}

We evaluated the baseline characteristics of our study cohort using descriptive statistics. We estimated the rate of non-aggressive cancer by applying the definition proposed by Buell, wherein the point at which cumulative lung cancer-specific survival curve no longer changes (ie, the slope approaches zero) can provide an estimate of cancer fatality among untreated cases. ${ }^{16}$ At this point, there are no additional lung cancer deaths (ie, progressive cancers) observed among a cohort of untreated patients with lung cancer, even after statistically adjusting for all deaths from other causes. Thus, surviving untreated lung cancer can be considered a 'non-aggressive' case. We determined the time point for estimating the rate of non-aggressive cancer based on the fatality curve for the entire cohort. Using these data, we selected the time point that corresponded to the derivative of the curve approaching zero. We generated survival curves by employing competing risks methods (conditional probability function $(\mathrm{CPF}))$ to estimate the long-term lung cancer-specific survival. $\mathrm{CPF}$ methods estimate the risk of lung cancer fatality conditional on an absence of death from competing causes. ${ }^{17}$ Competing risk estimation is preferable to the Kaplan-Meier method as the latter leads to biased disease-specific survival estimates when there is substantial comorbidity. This is especially important in the case of lung cancer, which in most cases is due to tobacco and occurs in the elderly, with a subsequent higher risk of cardiovascular and pulmonary comorbidity. Moreover, untreated patients are a group with a substantial burden of comorbidities.

To determine whether non-aggressive cancer varies according to baseline characteristics, we used stratified analyses according to sex, age, histology, tumour size and the presence of COPD. We tested for differences in survival according to patient characteristics using a z-test based on the asymptotically normal distribution of the conditional probability. ${ }^{18}$ All analyses were performed using SAS statistical software (SAS Institute, Cary, North Carolina, USA). This study was determined to be exempt from the Icahn School of Medicine at Mount Sinai institutional review board prior to study commencement.

\section{RESULTS}

We identified 2725 individuals in the SEER-Medicare files with untreated, clinically detected, stage I NSCLC, of which 528 did not have a claim for a chest CT, PET or mediastinoscopy, resulting in a final sample of 2197 patients. Approximately $33 \%$ of the sample was between the ages of 65 and 74, 50\% were between the ages of 75 and 84 and $17 \%$ were $>85$ years of age (table 1). The sample consisted of 51\% women, $80 \%$ Whites, 12\% Blacks, 3\% Hispanic and 5\% Other; 39\% were married. Approximately 30\%, 26\%, 24\% and 19\% belonged to the first (lowest), second, third and fourth (highest) income quartiles. In terms of size distribution, 4\%,17\%,22\%,14\% and 17\% had tumour sizes of $<15 \mathrm{~mm}, 15-24 \mathrm{~mm}, 25-34 \mathrm{~mm}, 35-44 \mathrm{~mm}$ and $\geq 45 \mathrm{~mm}$, respectively. We did not have tumour size data for $26 \%$ of individuals due to lack of data in SEER. Tumours' histology was 41\% adenocarcinoma, 40\% squamous cell, 6\% large cell and 13\% other. Approximately $47 \%$ of the cohort had COPD.

The CPF curve showing the risk of lung cancer fatality conditional on no death from competing causes in the total population is shown in figure 1 . The derivative of the conditional probability of lung cancer death approached zero at 100 months; this time point was used to assess non-aggressive cancer rates for all strata. The overall rate of non-aggressive cancer was $2.4 \%$, 95\% (CI): $1.0 \%$ to $3.8 \%$. Figures 2-6 demonstrate the CPF curves stratified by gender, tumour size, histology, the presence of COPD and age, respectively. The estimated rate of non-aggressive cancer was $1.9 \%$ (95\% CI: $0.0 \%$ to $4.9 \%$ ) for women and $2.4 \%(95 \% \mathrm{CI}: 0.7 \%$ to $4.1 \%)$ for men $(\mathrm{p}=0.84)$. When stratifying by tumour size, the estimated rate of non-aggressive 
Table 1 Baseline characteristics of patients with clinically detected, stage I untreated non-small cell lung cancer

\begin{tabular}{|c|c|c|}
\hline Characteristic, N (\%) & $\begin{array}{l}\text { Study } \\
\text { population } n=2197\end{array}$ & $95 \% \mathrm{Cl}$ \\
\hline \multicolumn{3}{|l|}{ Age (years) } \\
\hline $65-74$ & $725(33)$ & 31 to 35 \\
\hline $75-84$ & $1091(50)$ & 48 to 52 \\
\hline$>85$ & $381(17)$ & 16 to 19 \\
\hline Female & $1118(51)$ & 49 to 53 \\
\hline Married & $864(39)$ & 37 to 41 \\
\hline \multicolumn{3}{|l|}{ Race/ethnicity } \\
\hline White & $1767(80)$ & 79 to 82 \\
\hline Black & $254(12)$ & 10 to 13 \\
\hline Hispanic & $74(3)$ & 3 to 4 \\
\hline Other & $102(5)$ & 4 to 6 \\
\hline \multicolumn{3}{|l|}{ Median income } \\
\hline First quartile $^{*}$ & $666(30)$ & 28 to 32 \\
\hline Second quartile & $575(26)$ & 24 to 28 \\
\hline Third quartile & $528(24)$ & 22 to 26 \\
\hline Fourth quartile & $428(19)$ & 18 to 21 \\
\hline \multicolumn{3}{|l|}{ Diagnostic workup } \\
\hline CT chest & $2140(97)$ & (97 to 98 ) \\
\hline Positron emission tomography (PET) & $306(14)$ & (13 to 15$)$ \\
\hline Mediastinoscopy & $34(2)$ & (1 to 2 ) \\
\hline COPD & $1042(47)$ & 45 to 50 \\
\hline \multicolumn{3}{|l|}{ Year of diagnosis } \\
\hline 1992-1999 & $980(45)$ & 43 to 47 \\
\hline $2000-2009$ & $1217(55)$ & 53 to 57 \\
\hline \multicolumn{3}{|l|}{ Histology } \\
\hline Adenocarcinoma & $903(41)$ & 39 to 43 \\
\hline Squamous cell & $875(40)$ & 38 to 42 \\
\hline Large cell & $129(6)$ & 5 to 7 \\
\hline Other & $290(13)$ & 12 to 15 \\
\hline \multicolumn{3}{|l|}{ Tumour size $(\mathrm{mm})^{\dagger}$} \\
\hline$<15$ & $80(4)$ & 3 to 6 \\
\hline $15-24$ & $369(17)$ & 15 to 18 \\
\hline $25-34$ & $486(22)$ & 20 to 24 \\
\hline $35-44$ & $313(14)$ & 13 to 16 \\
\hline$>45$ & $377(17)$ & 16 to 19 \\
\hline
\end{tabular}

*Lowest income quartile

tFive hundred and seventy-two (26\%) with unknown tumour size.

cancer was $10.2 \%$ (95\% CI: $0.0 \%$ to $29.3 \%$ ), $2.1 \%$ (95\% CI: $0.0 \%$ to $9.2 \%), 4.9 \%$ (95\% CI: $0.0 \%$ to $10.3 \%), 1.8 \%$ (95\% CI: $0.0 \%$ to $5.2 \%$ ) and $0.0 \%$ (95\% CI: $0.0 \%$ to $1.0 \%)$ for tumour sizes $<15 \mathrm{~mm}, 15-24 \mathrm{~mm}, 25-34 \mathrm{~mm}, 35-44 \mathrm{~mm}$ and $\geq 45 \mathrm{~mm}$, respectively. In comparison with the smallest tumour sizes $(<15 \mathrm{~mm})$, the rates of non-aggressive cancers were not statistically significantly different for tumour sizes $15-24 \mathrm{~mm}(\mathrm{p}=0.36), 25-34 \mathrm{~mm}(\mathrm{p}=0.57), 35-44 \mathrm{~mm}(\mathrm{p}=0.38)$ and tumour sizes $>45 \mathrm{~mm}(\mathrm{p}=0.30)$. The estimated rates of non-aggressive cancers by histology were: adenocarcinoma, $4.5 \%$ (95\% CI: $1.2 \%$ to $7.7 \%$ ); squamous cell carcinoma, $0.7 \%$ (95\% CI: $0.0 \%$ to $3.1 \%$ ); large cell, $1.7 \%$ (95\% CI: $0.0 \%$ to

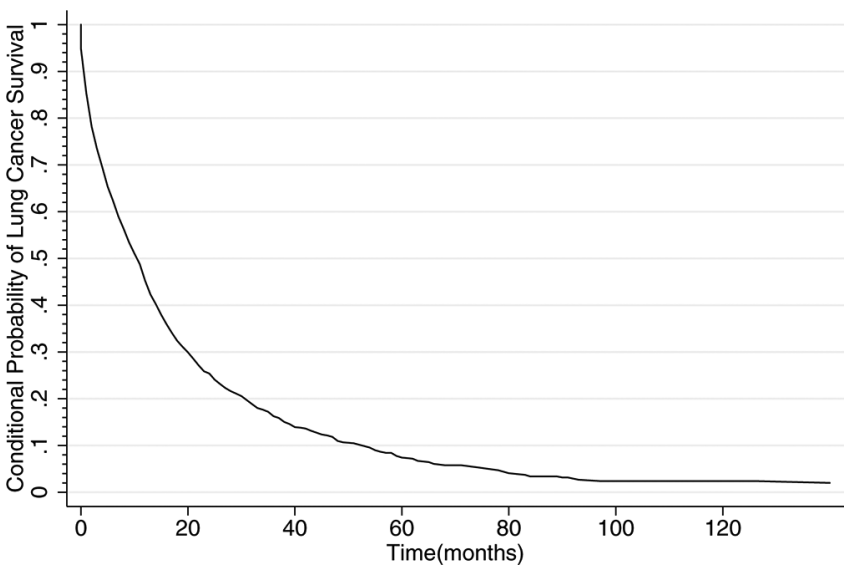

Figure 1 Conditional probability of lung cancer survival

7.9\%) and other, $2.2 \%$ (95\% CI: $0.0 \%$ to $5.4 \%)$ with $\mathrm{p}<0.005$ for all comparisons in reference to adenocarcinoma except for large cell carcinoma $(p=0.28)$. For individuals with and without COPD, the estimated rate of non-aggressive cancer was $1.2 \%$ (95\% CI: $0.0 \%$ to $3.3 \%$ ) and $3.3 \%$ (95\% CI: $1.3 \%$ to $5.3 \%$ ), $\mathrm{p}<0.05$, respectively. When stratifying by age, there was approximately $1.8 \%$ non-aggressive cancer ( $95 \%$ CI: $0.0 \%$ to $4.1 \%$ ) in individuals age $65-74,3.0 \%$ (95\% CI: 0.4 to $5.6, \mathrm{p}=0.02$ ) in individuals age $75-84$ and $0.9 \%$ (95\% CI: $0.0 \%$ to $4.1 \%$, $\mathrm{p}=0.43$ ) in individuals 85 years of age and older.

\section{DISCUSSION}

In our study of untreated, clinically detected stage I NSCLCs, we found overall low $(<10 \%)$ estimates of non-aggressive cancer regardless of age, sex, tumour histology and size and the presence of COPD, suggesting overdiagnosis of clinically detected NSCLC is relatively uncommon. These results suggest that most early stage lung cancer cases should be considered potentially aggressive and thus treated with a curative intent, a finding that is of particular importance in the management of incidentally found cancers. Biomarkers capable of predicting tumour behaviour are needed to minimise unnecessary treatment of potentially non-aggressive cancers.

Estimates of non-aggressive cancers among clinically detected cancers have yielded a wide range of values, depending on the method of estimation. In a study examining the outcomes of individuals with early stage lung cancer who were not surgically resected, the rate of non-aggressive cancers was estimated to be

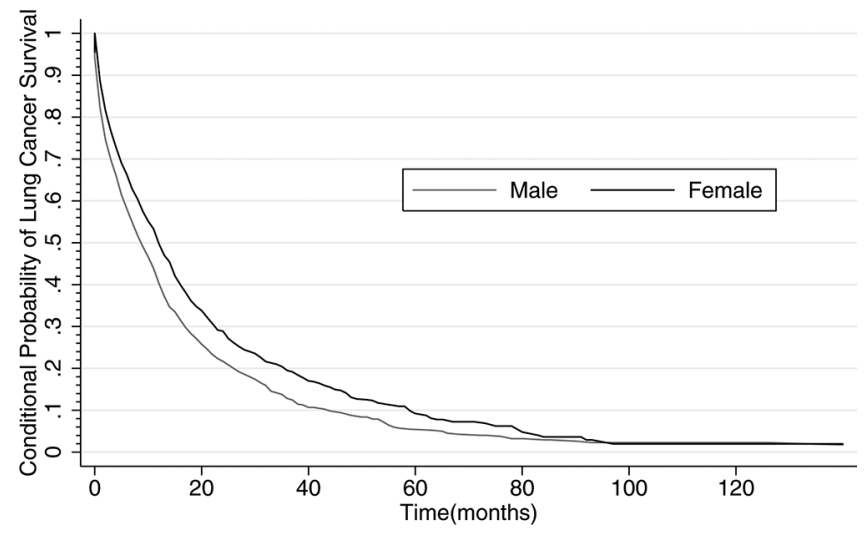

Figure 2 Conditional probability of lung cancer survival according to gender 


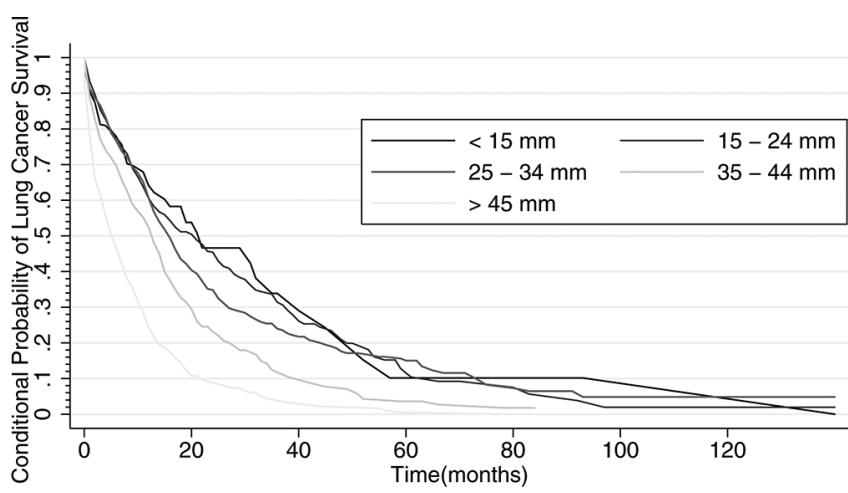

Figure 3 Conditional probability of lung cancer survival accor ding to tumour size

$17 \%$, but a majority of patients $(80 \%)$ did receive some form of treatment (chemotherapy or radiation therapy), and $10 \%$ of the cancers were of the small cell subtype. ${ }^{19}$ In a separate study of SEER data, the rate of non-aggressive cancers was estimated using the Kaplan-Meier method to control for non-cancer deaths as opposed to using a more valid competing risks framework. ${ }^{20}$ In a comprehensive review of studies that have examined growth rate, complementary DNA composition and autopsy analysis, along with trial data (primarily of chest radiography studies), the rate of non-aggressive cancers ranged from $11 \%$ to $25 \%$ but was deemed to be difficult to accurately quantify, although of clinical significance in relation to screening. ${ }^{21}$

The rate of overdiagnosis among screen-detected cancers is likely to be different as screening with CT may unveil small, slowly progressive cancers that are biologically dissimilar. Data from the NLST included 8 years of follow-up, yielding a rate of overdiagnosis, defined as the excess NSCLCs detected by CT divided by all CT screen-detected NSCLCs, of 22\%, an estimate that will likely decline as more follow-up is accrued. ${ }^{7}$ Modelling studies that have been extensively used to study the comparative effectiveness of various screening scenarios and have informed recommendations by the United States Preventive Services Task Force (USPSTF) can also estimate the extent of overdiagnosis associated with screening. ${ }^{22}$ Modelling studies have found an average overdiagnosis rate of $11.9 \%$ in the age range recommended by the USPSTF. In light of different recommended ages at which to screen for lung cancer by different organisations, overdiagnosis may serve as useful metric by which to assess the benefits and harms of lung cancer screening, as in their analysis,

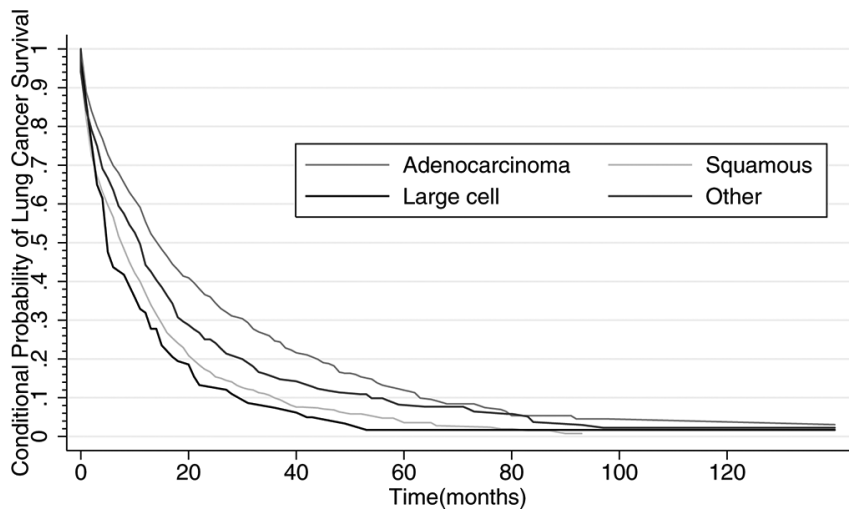

Figure 4 Conditional probability of lung cancer survival according to tumour histology

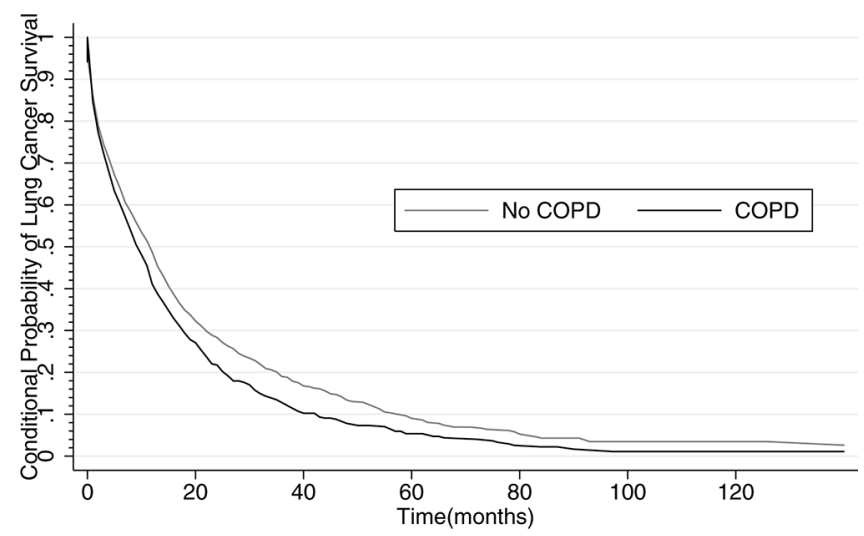

Figure 5 Conditional probability of lung cancer survival according to the presence of COPD

stopping screening at earlier age led to fewer overdiagnosed cases per lung cancer death averted. ${ }^{23}$

Our findings must be considered in light of certain strengths and limitations of our study. First, our analysis consists of stage I NSCLCs largely in individuals $>65$ years of age, diagnosed prior to the advent of widespread lung cancer screening, and thus represent cancers detected clinically. It is unlikely that we can extrapolate our findings to younger individuals or to stage I NSCLCs detected through screening. However, we expect that the smaller cancers $(<15 \mathrm{~mm})$ were likely asymptomatic and detected on imaging incidentally and thus similar to cancers diagnosed by screening. Additionally, analyses limited to individuals who underwent a baseline chest CT showed similar rates of overdiagnosis. Second, we were limited by the noted cause of death as reported by the death certificate data, which is subject to inaccuracies. However, studies assessing the validity of death certificate data suggest that the use of cause of death data obtained from death certificates was not significantly different from cause of death determined by a mortality review committee. ${ }^{24}$ Third, we did not have information on reason for lack of treatment in these individuals (either poor medical fitness for surgery or surgical inoperability), as these individuals may have a different risk of lung cancer death than the group that received treatment and may give a different estimate of the rate of non-aggressive cancers. Additionally, we were limited by a lack of information on smoking history, a variable not captured in SEER-Medicare, and incomplete values of tumour size, due to missing information in SEER. Finally, we were limited by an inability to assess the rate of non-aggressive cancers in minimally

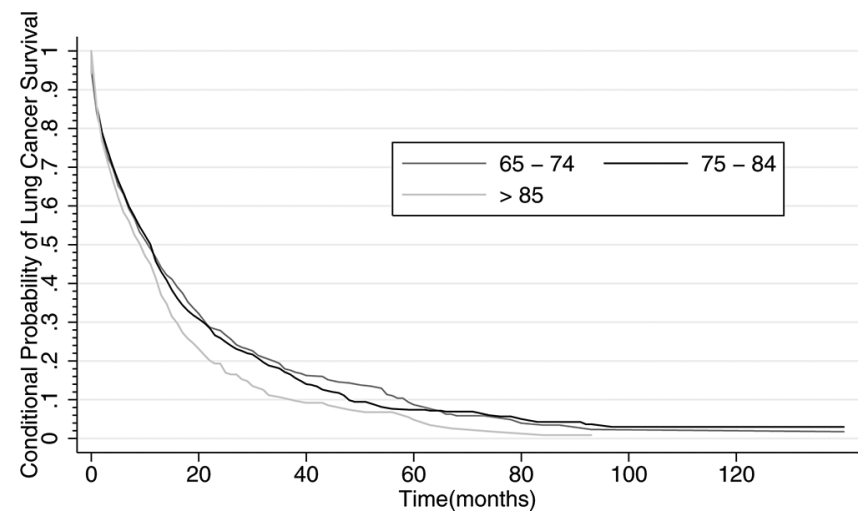

Figure 6 Conditional probability of lung cancer survival according to age

Kale MS, et al. Thorax 2018;73:459-463. doi:10.1136/thoraxjnl-2017-210309 
invasive adenocarcinoma and adenocarcinoma in situ, as these were previously classified within the adenocarcinoma subtype. However, our study had several strengths, notably the use of nationally representative data with large numbers of individuals as well as precise estimation and analyses of subgroups. Additionally, we were able to construct our survival curves using long-term follow-up information that allowed for the accurate assessment of curve plateau. Finally, with our use of competing risks methods, we were able to appropriately adjust for deaths from other causes leading to overdiagnosis of estimates with limited bias.

In our population-based assessment of stage I NSCLCs, we found low rates of non-aggressive cancers overall as well as in stratified analyses, after adjusting for competing risks. These results are useful for the management of clinically detected, early stage cancers and suggest that most cases should be considered for curative resection.

Acknowledgements This study used the linked Surveillance, Epidemiology, and End Results (SEER)-Medicare Database. The interpretation and reporting of these data are the sole responsibility of the authors. The authors acknowledge the efforts of the Applied Research Program, National Cancer Institute; the Office of Research, Development and Information, Centers for Medicare and Medicaid Services; Information Management Services (IMS), Inc and SEER Program tumour registries in the creation of the SEER-Medicare Database. The authors would also like to thank Joey Kong, PhD, for his thoughtful comments.

Contributors Study concept and design: MSK, KS and JPW. Acquisition, analysis or interpretation of data: MSK, GM, KS and JPW. Drafting of the manuscript: MSK. Critical revision of the manuscript for important intellectual content: GM, KS and JPW.

Competing interests JPW is a member of the research board of EHE International, has received consulting honorarium from Quintiles, AstraZeneca and Merck and a research grant from Aventis Pharmaceutical and Quorum. KS has received honorarium from Gilead.

Ethics approval Icahn School of Medicine Institutional review board.

Provenance and peer review Not commissioned; externally peer reviewed.

Data sharing statement MSK had full access to all the data in the study and takes responsibility for the integrity of the data and the accuracy of the data analysis.

(c) Article author(s) (or their employer(s) unless otherwise stated in the text of the article) 2018. All rights reserved. No commercial use is permitted unless otherwise expressly granted.

\section{REFERENCES}

1 Welch HG, Black WC. Overdiagnosis in cancer. J Natl Cancer Inst 2010;102:605-13.

2 Esserman $\mathrm{L}$, Thompson IM, Reid B, et al. Addressing overdiagnosis and overtreatment in cancer: a prescription for change. Lancet Oncol 2014;15:e234-e242.
3 Marcus PM, Prorok PC, Miller AB, et al. Conceptualizing overdiagnosis in cancer screening. J Natl Cancer Inst 2015;107:djv014.

4 Eddy DM. Screening for lung cancer. Ann Intern Med 1989;111:232-7.

5 Kubik A, Parkin DM, Khlat M, et al. Lack of benefit from semi-annual screening for cancer of the lung: follow-up report of a randomized controlled trial on a population of high-risk males in Czechoslovakia. Int J Cancer 1990;45:26-33.

6 de Koning HJ, Meza R, Plevritis SK, et al. Benefits and harms of computed tomography lung cancer screening strategies: a comparative modeling study for the U.S. Preventive Services Task Force. Ann Intern Med 2014;160:311-20.

7 Patz EF, Pinsky P, Gatsonis C, et al. Overdiagnosis in low-dose computed tomography screening for lung cancer. JAMA Intern Med 2014;174:269-74.

8 Infante M, Cavuto S, Lutman FR, et al. Long-term follow-up results of the DANTE Trial, a randomized study of lung cancer screening with spiral computed tomography. Am J Respir Crit Care Med 2015;191:1166-75.

9 Patz EF. Lung cancer screening, overdiagnosis bias, and reevaluation of the Mayo Lung Project. J Natl Cancer Inst 2006;98:724-5.

10 Warren JL, Klabunde CN, Schrag D, et al. Overview of the SEER-Medicare data: content, research applications, and generalizability to the United States elderly population. Med Care 2002;40(8 Suppl):IV-3-18.

11 Institute NC. Surveillance, epidemiology, and end results: NIH. 2012 http://seer.cancer. gov/about/factsheets/SEER_brochure.pdf (accessed Mar 2012).

12 Klabunde CN, Potosky AL, Legler JM, et al. Development of a comorbidity index using physician claims data. J Clin Epidemiol 2000;53:1258-67.

13 Klabunde CN, Legler JM, Warren JL, et al. A refined comorbidity measurement algorithm for claims-based studies of breast, prostate, colorectal, and lung cancer patients. Ann Epidemiol 2007;17:584-90.

14 Detterbeck FC, Boffa DJ, Tanoue LT. The new lung cancer staging system. Chest 2009;136:260-71.

15 Bach PB, Guadagnoli E, Schrag D, et al. Patient demographic and socioeconomic characteristics in the SEER-Medicare database applications and limitations. Med Care 2002;40(8 Suppl):IV-19-25.

16 Buell PE. The importance of tumor size in prognosis for resected bronchogenic carcinoma. J Surg Oncol 1971;3:539-51.

17 Pintilie M. Competing risks: a practical perspective. Chichester, England; Hoboken, NJ: John Wiley \& Sons, 2006; xiii:224.

18 JKaM M. Survival analysis: techniques for censored and truncated data. New York, NY: Springer, 1997.

19 Sobue T, Suzuki T, Matsuda M, et al. Survival for clinical stage I lung cancer not surgically treated. comparison between screen-detected and symptom-detected cases. Cancer 1992;69:685-92.

20 Henschke Cl, Wisnivesky JP, Yankelevitz DF, et al. Small stage I cancers of the lung: genuineness and curability. Lung Cancer 2003;39:327-30.

21 Reich JM. A critical appraisal of overdiagnosis: estimates of its magnitude and implications for lung cancer screening. Thorax 2008;63:377-83.

22 Moyer VA; U.S. Preventive Services Task Force. Screening for lung cancer: U.S. Preventive Services Task Force recommendation statement. Ann Intern Med 2014:160:330-338-8.

23 Han SS, Ten Haaf K, Hazelton WD, et al. The impact of overdiagnosis on the selection of efficient lung cancer screening strategies. Int I Cancer 2017; 140:2436-43.

24 Doria-Rose VP, Marcus PM. Death certificates provide an adequate source of cause of death information when evaluating lung cancer mortality: an example from the Mayo Lung Project. Lung Cancer 2009;63:295-300. 